OPEN ACCESS

Edited by:

Anna Sebestyén, Semme/weis University,

Hungary

*Correspondence:

Maher Kurdi

Ahkurdi@kau.edu.sa

Received: 12 February 2021 Accepted: 07 April 2021

Published: 29 April 2021

Citation:

Kurdi M, Shafique Butt N, Baeesa S, Alghamdi B, Maghrabi $Y$, Bardeesi A,

Saeedi R, Al-Sinani T, Alghanmi N,

Bari MO, Samkari A and Lary Al (2021)

The Impact of IDH1 Mutation and MGMT Promoter Methylation on

Recurrence-Free Interval in

Glioblastoma Patients Treated With

Radiotherapy and

Chemotherapeutic Agents.

Pathol. Oncol. Res. 27:1609778.

doi: 10.3389/pore.2021.1609778

\section{The Impact of IDH1 Mutation and MGMT Promoter Methylation on Recurrence-Free Interval in Glioblastoma Patients Treated With Radiotherapy and Chemotherapeutic Agents}

\begin{abstract}
Maher Kurdi ${ }^{1 *}$, Nadeem Shafique Butt ${ }^{2}$, Saleh Baeesa ${ }^{3}$, Badrah Alghamdi ${ }^{4}$, Yazid Maghrabi $^{5}$, Anas Bardeesi ${ }^{5}$, Rothaina Saeedi ${ }^{3}$, Taghreed Al-Sinani ${ }^{6}$, Najla Alghanmi ${ }^{7}$, Mohammed O. Bari ${ }^{7}$, Alaa Samkari ${ }^{8}$ and Ahmed I. Lary ${ }^{9}$

${ }^{1}$ Department of Pathology, Faculty of Medicine in Rabigh, King Abdulaziz University, Jeddah, Saudi Arabia, ${ }^{2}$ Department of Family and Community Medicine, Faculty of Medicine in Rabigh, King Abdulaziz University, Jeddah, Saudi Arabia, ${ }^{3}$ Division of Neurosurgery, Faculty of Medicine, King Abdulaziz University, Jeddah, Saudi Arabia, ${ }^{4}$ Department of Physiology, Faculty of Medicine, King Abdulaziz University, Jeddah, Saudi Arabia, ${ }^{5}$ Department of Neuroscience, King Faisal Specialist Hospital, Jeddah, Saudi Arabia, ${ }^{6}$ Department of Surgery, Division of Neurosurgery, King Fahad General Hospital, Jeddah, Saudi Arabia, ${ }^{7}$ Department of Pathology, King Abdulaziz University Hospital, Jeddah, Saudi Arabia, ${ }^{8}$ Department of Pathology and Laboratory Medicine, King Saud Bin Abdulaziz University for Health Science, Jeddah, Saudi Arabia, ${ }^{9}$ Section of Neurosurgery, Department of Surgery, King Abdulaziz Medical City, Jeddah, Saudi Arabia
\end{abstract}

The aim of this study is to investigate the relationship between isocitrate dehydrogenase-1 (IDH1) mutation and $\mathrm{O}^{6}$-methylguanine-DNA methyltransferase (MGMT) promoter methylation with recurrence-free interval in glioblastoma patients treated with chemoradiotherapies. Clinical data were collected from 82 patients with totally resected glioblastoma and treated with adjuvant therapies from 2014 to 2019. IDH1 mutation was assessed by immunohistochemistry and MGMT promoter methylation was assessed by different sequencing methods. IDH1 mutation was present in 32 cases and 50 cases were IDH1 wildtype; 54 and 28 patients had unmethylated and methylated MGMT promoter, respectively, Of the 82 patients, 62 patients received chemoradiotherapy while 20 patients only received radiation. Approximately, $61 \%$ of patients had a tumor recurrence after 1 year, and 39\% showed a recurrence before 1 year of treatment. There was no significant relationship between IDH1 mutation and MGMT promoter methylation ( $p$-value $=0.972$ ). Patients with $I D H 1$ mutation and their age $<50$ years showed a significant difference in recurrence-free interval $(p$-value $=0.014)$. Difference in recurrence-free interval was also statistically observed in patients with unmethylated MGMT promoter and treated with chemoradiotherapies $(p$-value $=0.031)$, by which they showed a late tumor recurrence ( $p$-value $=0.016$ ). This revealed that IDH1 mutation and MGMT methylation are independent prognostic factors in glioblastoma. Although IDH1-mutant glioblastomas showed late tumor recurrence in patients less than 50 years old, the type of treatment modalities may not show additional beneficial outcome. Patients with unmethylated MGMT and IDH1 mutation, treated with different chemoradiotherapies, showed a late tumor recurrence.

Keywords: glioblastoma, chemotherapy, IDH1 mutation, MGMT promoter methylation, radiotherapy 


\section{INTRODUCTION}

Glioblastoma is the most aggressive primary malignant brain tumor in adults [1]. The current standard treatment for glioblastoma after surgical resection is combined radiotherapy and chemotherapy using temozolamide (TMZ) or TMZ combined with other chemotherapeutic agents. Despite these treatments, glioblastoma remains a fatal disease and the overall survival is approximately 14.6 months within five years [2, 3]. However, the current treatment strategies are considered palliative, with the aim being to improve patient survival and maintain a good quality of life. Several biomarkers are still used in clinical practice to improve the diagnostic and prognostic status of glioblastoma. One of these markers are $\mathrm{IDH} 1^{\mathrm{R} 132 \mathrm{H}}$ mutation and $M G M T$ gene promoter methylation. The IDH1 gene, which encodes cytosolic NADP + -dependent isocitrate dehydrogenase, was shown to correlate with outcome of patients with glioblastoma [4-6]. The $M G M T$, which is a DNA repair protein that removes alkyl groups from several residues particularly the O6-position of guanine, is considered the most relevant for the action of an extensively used TMZ [7, 8]. Because IDH1 ${ }^{\mathrm{R} 132 \mathrm{H}}$ mutation, is the most common variant mutation detected in most of cancers, particularly glioblastoma, we used specific monoclonal antibody (IMAB-1) targeted $\mathrm{R} 131 \mathrm{H}$ residue.

The relationship of MGMT promoter methylation with $I D H 1$ mutation, treatment modalities and survival rates showed controversial results. Some studies revealed that glioblastomas with methylated $M G M T$ promoter were more sensitive to chemotherapeutic agent (TMZ) resulting in a better survival rate [9-11], while other studies revealed that MGMT promoter methylation was not associated with better overall survival (OS) $[12,13]$. Combs et al. also found a none-favourable OS in a group of 160 patients with glioblastoma treated with radiotherapy and TMZ. In contrast, Millward et al. demonstrated that the combination of methylated MGMT promoter and IDH1 mutation was associated with considerably longer OS in a series of chemoradiotherapy-treated glioblastoma tumors [14]. Despite this controversy, both biomarkers are still considered prognostic factors in patients with glioblastoma.

In this study, we evaluated the impact of MGMT promoter methylation and $I D H 1$ mutation on the recurrence-free interval in patients with glioblastoma treated with different treatment modalities (radiotherapy and different types of chemotherapies). The chemotherapy protocol included temozolomide (TMZ) alone, or TMZ and other adjuvant therapies included (bevacizumab, irinotecan, lomustine, and etoposide). This study provided an additional justification to the previous controversial results in the literature. To our knowledge, this study is considered as a first research of such type performed in Saudi Arabian patients.

\section{MATERIALS AND METHODS}

\section{Patients and Stratification}

The study included 82 patients with completely resected glioblastoma from two medical institutions in Saudi Arabia in the period of 2014-2019. This study was approved by the National Biomedical Ethics Committee at King Abdulaziz University (HA-02-J-008) under a general ethical report. All patients underwent complete surgical resection of the tumor followed by a standard protocol of chemoradiotherapy. The histological diagnosis was made based on classification of the World Health Organization (WHO). Clinical data were retrieved from the hospital records and included patient age at diagnosis, gender, post-operative adjuvant therapies, MGMT methylation profile, and IDH1 results. The patients were stratified into different groups based on the adjuvant therapies (Figure 1). Standard radiotherapy of a total dose of $60 \mathrm{~Gy}$ and TMZ $\left(150-200 \mathrm{mg} / \mathrm{m}^{2}\right.$ for $6-12$ cycles) was given to all patients at the time of management. The chemotherapy protocol included temozolomide (TMZ) alone, or TMZ and other adjuvant therapies included (bevacizumab, irinotecan, lomustine, and etoposide). All patients enrolled in this study have passed away. Those who had late recurrence died because of other comorbidities. The only limitation should be clarified that the total number of cases analyzed in this study is still considered small. Despite this limitation, this is the first study in Saudi Arabia that explore the impact of MGMT methylation and IDH1 on tumour recurrence in patients receiving different type of treatment modalities.

\section{Assessment of IDH1 Mutation}

IDH1 mutation was evaluated by immunohistochemistry. AntiIDH1 ${ }^{\mathrm{R} 132 \mathrm{H}}$ mouse monoclonal antibody (clone H09) was used to identify wild-type and mutant IDH1 in formalin-fixed paraffin-embedded (FFPE) sections using a BenchMark XT automated staining system from Ventana. Sections in which $>10 \%$ of tumor cells positively stained were defined as IDH1 mutant.

\section{Assessment of MGMT Promoter Methylation}

MGMT promoter methylation was assessed in the hospitals at time of histopathological diagnosis by using two different techniques: methylation-specific polymerase chain reaction (MSP) or pyrosequencing method using Qiagen.

First method used: Qualitative methylation-specific PCR (MSP). The regions were selected from which DNA could be extracted. DNA extraction was performed using the QIAamp DNA FFPE tissue kit according to the manufacturer's instructions. DNA quantity and quality were determined using a NanoDrop spectrophotometer at A260/A280 and A260/A230. The concentration of DNA samples was normalized to $50 \mathrm{ng}$ and bisulfite-converted using the EpiTect Bisulfite Kit (Qiagen) according to the manufacturer's instructions. The forward and reverse primers targeting methylated and unmethylated exon 1 of the human MGMT gene correspond to those described by Esteller et al. [15]. The PCR Kit used was HotStarTaq plus DNA polymerase (Qiagen). Thermal cycling on a Veriti thermal cycler included an initial step at $95^{\circ} \mathrm{C}$ for 2 min followed by 40 cycles of $30 \mathrm{~s}$ at $94^{\circ} \mathrm{C}, 30 \mathrm{~s}$ at $52^{\circ} \mathrm{C}$, and $30 \mathrm{~s}$ at $72^{\circ} \mathrm{C}$ for $10 \mathrm{~min}$. The 


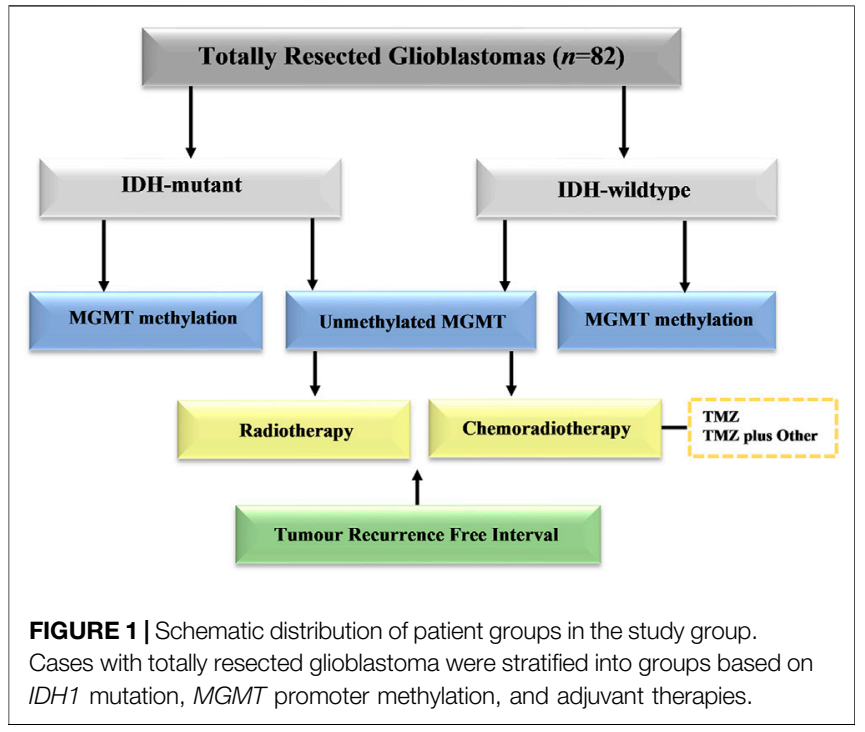

PCR products were visualized on $8 \%$ non-denaturing polyacrylamide gels and stained with ethidium bromide. Samples having only methylated PCR products and samples having both methylated and non-methylated PCR products were both scored as methylation positive.

Second method used: Pyrosequencing technique. The MGMT pyro Kit from Qiagen has been used for quantitative measurement of methylation at four $\mathrm{CpG}$ sites in exon 1 of human MGMT gene (genomic sequence on chromosome 10 from $131,265,519$ to $131,265,537$ sequencing from 72 to 90 on MGMT mRNA. DNA extraction was performed using the QIAamp DNA FFPE tissue kit according to the manufacturer's instructions. DNA quantity and quality were determined using a NanoDrop spectrophotometer. The concentration of DNA samples was normalized to $50 \mathrm{ng}$ and bisulfite-converted using the EpiTect Bisulfite Kit (Qiagen) according to the manufacturer's recommendation. The Thera screen MGMT PyroKit and PyroMark Q24 system were both used to assess the methylation status. DNA was amplified by PCR while single-stranded DNA was prepared, sequenced, and finally analyzed on PyroMark Q24. The methylated control DNA was included in the kit as a positive control for PCR and sequencing reaction. The procedure corresponds to what has been described by Pangopoulos et al [16].

\section{Statistical Methods}

Data are described as frequencies and percentages. Pearson's Chi-Square and Fisher's Exact tests were used to explore the association of IDH1 mutation, MGMT promoter methylation status, and chemotherapies with various study factors. Kaplan-Meier curves and log-rank test were used to compare the distribution of recurrence-free interval. Recurrence-free interval was defined as the period from the beginning of adjuvant therapy after surgical resection to the possible first date of recurrence. In survival analysis, the term "event" is usually used to measure the occurrence of an event of
TABLE 1 | Patient and tumor characteristics of the study group $(n=82)$.

\begin{tabular}{lc} 
Characteristic & $\boldsymbol{n}$ (\%) \\
\hline Age & \\
Mean & 48.4 \\
Age group & \\
$\quad<50$ years & $32(39.0 \%)$ \\
$\geq 50$ years & $50(61.0 \%)$ \\
Gender & \\
Female & $34(41.5 \%)$ \\
Male & $48(58.5 \%)$ \\
IDH1 mutation status & \\
IDH1 mutant & $32(39.0 \%)$ \\
IDH1 wildtype & $50(61.0 \%)$ \\
MGMT promoter methylation & \\
Methylation & $28(34.1 \%)$ \\
Unmethylated & $54(65.9 \%)$ \\
Adjuvant therapy & \\
Radiotherapy & $20(24.4 \%)$ \\
Radiotherapy and chemotherapy & $62(75.6 \%)$ \\
Chemotherapy & \\
Temozolomide & \\
Temozolomide plus others & $45(72.5 \%)$ \\
Recurrence & $17(27.5 \%)$ \\
Before 1 year & \\
After 1 year & $32(39.0 \%)$ \\
& $50(61.0 \%)$
\end{tabular}

interest (such as death, recurrence, and recovery). In our study, the event of interest is "recurrence." The "number of cases at risk" defines how many cases will be at risk in the end of a specified time point. Being "at risk" clarifies that the patient has not had a recurrence before a time and is not censored before or at time. Moreover, the number of events" measures that number of cases for which an event of interest is observed. All statistical analyses in this study were performed using IBM SPSS1 ver. 24 statistical software programs (SPSS Inc. Chicago, IL, United States).

\section{RESULTS}

A total of 82 glioblastoma patients with complete resection and who received treatment were included in this study. The patient and tumor characteristics of the study group are listed in Table 1. The mean patient age was 48 years old ( $<50$ years old, $n=32$; $\geq 50$ years old, $n=50$ ), and $58.5 \%$ of the study group was male $(n=48)$. IDH1 mutation was found in 32 cases $(39 \%)$ and the remaining 50 cases $(61 \%)$ were $I D H 1$ wildtype. The MGMT promoter was methylated in 28 patients (34.1\%) and unmethylated in 54 cases $(65.9 \%)$. After complete tumor resection, 62 patients $(75.6 \%)$ received chemoradiotherapy while 20 patients $(24.4 \%)$ only received radiotherapy. Among those who received chemoradiotherapy, approximately $72.5 \%(n=45)$ received TMZ alone while $27.5 \%(n=17)$ received TMZ along with other chemotherapies included bevacizumab, irinotecan, lomustine, or etoposide. Approximately 61\% $(n=50)$ had tumor recurrence after 1 year, while $39 \%(n=32)$ showed recurrence before 1 year of the adjuvant therapies. 
TABLE 2 | Relationship between IDH1 mutation and MGMT promoter methylation in glioblastoma patients.

\begin{tabular}{|c|c|c|c|c|}
\hline & $\begin{array}{c}\text { MGMT } \\
\text { methylation }(n=28)\end{array}$ & $\begin{array}{l}\text { Unmethylated } \\
\text { MGMT }(n=54)\end{array}$ & Total $(n=82)$ & $p$-value \\
\hline$I D H$ status & & & & $0.972^{a}$ \\
\hline IDH1 mutant & 11.0 (39.3\%) & 21.0 (38.9\%) & $32.0(39.0 \%)$ & \\
\hline IDH1 wildtype & $17.0(60.7 \%)$ & $33.0(61.1 \%)$ & $50.0(61.0 \%)$ & \\
\hline
\end{tabular}

Data are shown as $\mathrm{n}(\%)$

aPearson's Chi-squared test.

TABLE 3 | The relationship between age, IDH1 mutation, and MGMT promoter methylation in glioblastoma patients and one-year recurrence-free interval.

\begin{tabular}{|c|c|c|c|c|c|c|c|c|}
\hline \multirow[b]{3}{*}{ Age } & \multirow[b]{3}{*}{$<50$ years } & \multirow[b]{3}{*}{ IDH1 status } & \multirow[b]{3}{*}{ IDH1 mutant } & \multicolumn{4}{|c|}{ Recurrence-free interval } & \multirow{3}{*}{$\frac{\boldsymbol{p} \text {-value } \mathrm{e}^{\mathrm{a}, \mathrm{b}}}{0.014^{\mathrm{a}}}$} \\
\hline & & & & \multicolumn{2}{|c|}{$<1$ year } & \multicolumn{2}{|c|}{$\geq 1$ year } & \\
\hline & & & & 3 & $(17.6 \%)$ & 14 & (82.4\%) & \\
\hline & & & IDH1 wildtype & 9 & (60.0\%) & 6 & (40.0\%) & \\
\hline & $\geq 50$ years & & IDH1 mutant & 3 & (20.0\%) & 12 & (80.0\%) & $0.059^{a}$ \\
\hline & & & IDH1 wildtype & 17 & (48.6\%) & 18 & (51.4\%) & \\
\hline \multirow[t]{4}{*}{ Age } & $<50$ years & MGMT status & Methylation & 5 & (50.0\%) & 5 & $(50.0 \%)$ & $0.438^{b}$ \\
\hline & & & Unmethylated & 7 & (31.8\%) & 15 & (68.2\%) & \\
\hline & $\geq 50$ years & & Methylation & 5 & (27.8\%) & 13 & $(72.2 \%)$ & $0.188^{a}$ \\
\hline & & & Unmethylated & 15 & (46.9\%) & 17 & (53.1\%) & \\
\hline \multirow[t]{4}{*}{ IDH1 status } & IDH1 mutant & Adjuvant therapy & Radiation & 1 & (33.3\%) & 2 & $(66.7 \%)$ & $0.476^{b}$ \\
\hline & & & Chemoradiotherapy & 5 & (17.2\%) & 24 & (82.8\%) & \\
\hline & IDH1 wildtype & & Radiation & 11 & (64.7\%) & 6 & (35.3\%) & $0.197^{\mathrm{a}}$ \\
\hline & & & Chemoradiotherapy & 15 & (45.5\%) & 18 & (54.5\%) & \\
\hline \multirow[t]{4}{*}{ MGMT status } & Methylated & Adjuvant therapy & Radiation & 1 & (50.0\%) & 1 & (50.0\%) & $0.999^{b}$ \\
\hline & & & Chemoradiotherapy & 9 & (34.6\%) & 17 & $(65.4 \%)$ & \\
\hline & Unmethylated & & Radiation & 11 & $(61.1 \%)$ & 7 & $(38.9 \%)$ & $0.031^{a}$ \\
\hline & & & Chemoradiotherapy & 11 & (30.6\%) & 25 & (69.4\%) & \\
\hline \multirow[t]{4}{*}{ IDH1 status } & IDH1 mutant & Chemotherapy & $\mathrm{TMZ}$ & 5 & (23.8\%) & 16 & (76.2\%) & $0.283^{b}$ \\
\hline & & & TMZ plus others & 0 & (0.0\%) & 8 & $(100.0 \%)$ & \\
\hline & IDH1 wildtype & & $\mathrm{TMZ}$ & 15 & $(60.0 \%)$ & 10 & $(40.0 \%)$ & $0.118^{b}$ \\
\hline & & & TMZ plus others & 2 & (22.2\%) & 7 & (77.8\%) & \\
\hline \multirow[t]{4}{*}{ MGMT status } & Methylated & Chemotherapy & $\mathrm{TMZ}$ & 8 & $(42.1 \%)$ & 11 & $(57.9 \%)$ & $0.666^{b}$ \\
\hline & & & TMZ plus others & 2 & (25.0\%) & 6 & (75.0\%) & \\
\hline & Unmethylated & & $\mathrm{TMZ}$ & 12 & (44.4\%) & 15 & (55.6\%) & $0.016^{b}$ \\
\hline & & & TMZ plus others & 0 & $(0 \%)$ & 9 & (100.0\%) & \\
\hline
\end{tabular}

*Data are shown as $n(\%)$.

${ }^{a} \mathrm{p}$-Values of Chi-Square test.

${ }^{b} \mathrm{p}$-Values of Fisher's Exact test.

\section{IDH1 Mutation and MGMT Methylation in Glioblastoma Patients}

Although wildtype $I D H 1$ was frequently found in glioblastoma patients with unmethylated $M G M T$ promoter, there was no significant relationship between IDH1 mutation and MGMT promoter methylation in the study group $(p$-value $=0.972)($ Table 2$)$.

\section{Relationship of Age and IDH1 Mutation or MGMT Methylation Status With Recurrence-Free Interval in Glioblastoma Patients}

Among patients less than 50 years of age, a significant difference was observed in recurrence-free interval between glioblastomas with IDH1 mutation and wildtype IDH1 $(p$-value $=0.014)$
(Table 3). These results indicated that IDH1 mutant cases showed a late recurrence rate compared with those with wildtype IDH1 (Figure 2A). In contrast, patients over 50 years of age did not show any significant difference in recurrence-free interval, regardless of IDH1 status. There was no significant difference in recurrence-free interval among glioblastoma patients based on age and MGMT promoter methylation status.

\section{Relationship of IDH1 Mutation or MGMT Promoter Methylation and Adjuvant Therapies With Recurrence-Free Interval in Glioblastoma Patients}

There was no significant difference in tumor recurrence among patients treated with any adjuvant therapy protocol regardless of 


\section{A \\ Distribution of IDH1 mutation and Recurrence Rate among Patients' age $<50$-years}

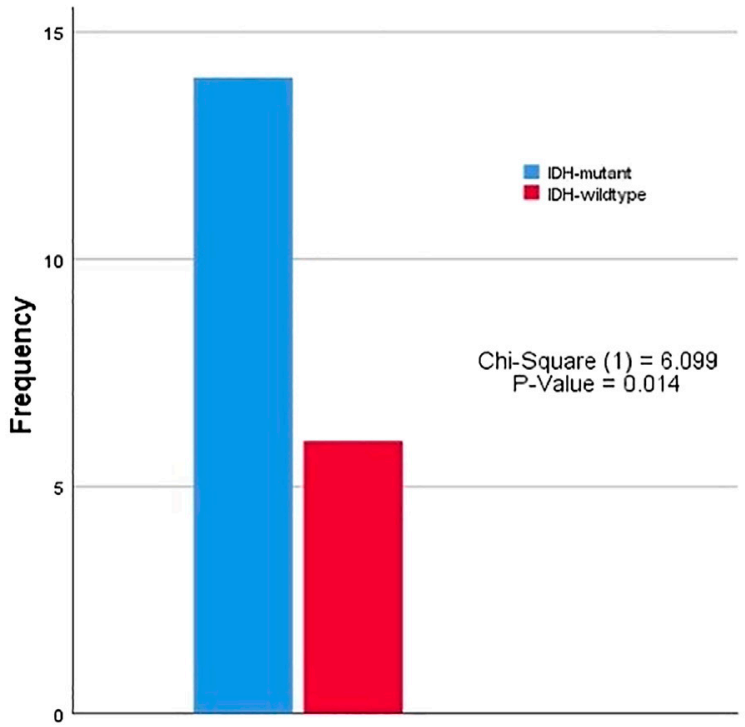

Tumour Recurrence $=>$ 1-year
B

\section{Distribution of IDH1 mutation among Unmethylated MGMT Patients}

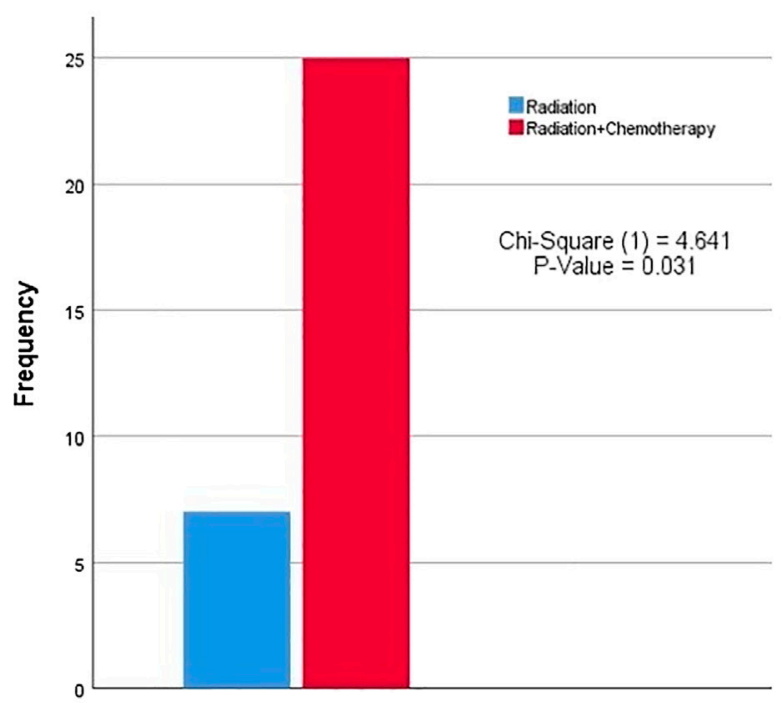

Tumour Recurrence $=>$ 1-year

FIGURE 2 | The relationship between IDH1-mutation status and MGMT promoter methylation with patients age, recurrence rate or type of chemotherapies. (A) Among patients younger than 50 years of age, patients with IDH1 mutation had a late recurrence after one year compared with patients with wildtype IDH1, (B) patients with unmethylated MGMT promoter had a recurrence after 1 year of chemoradiotherapy compared with those who received only radiotherapy.
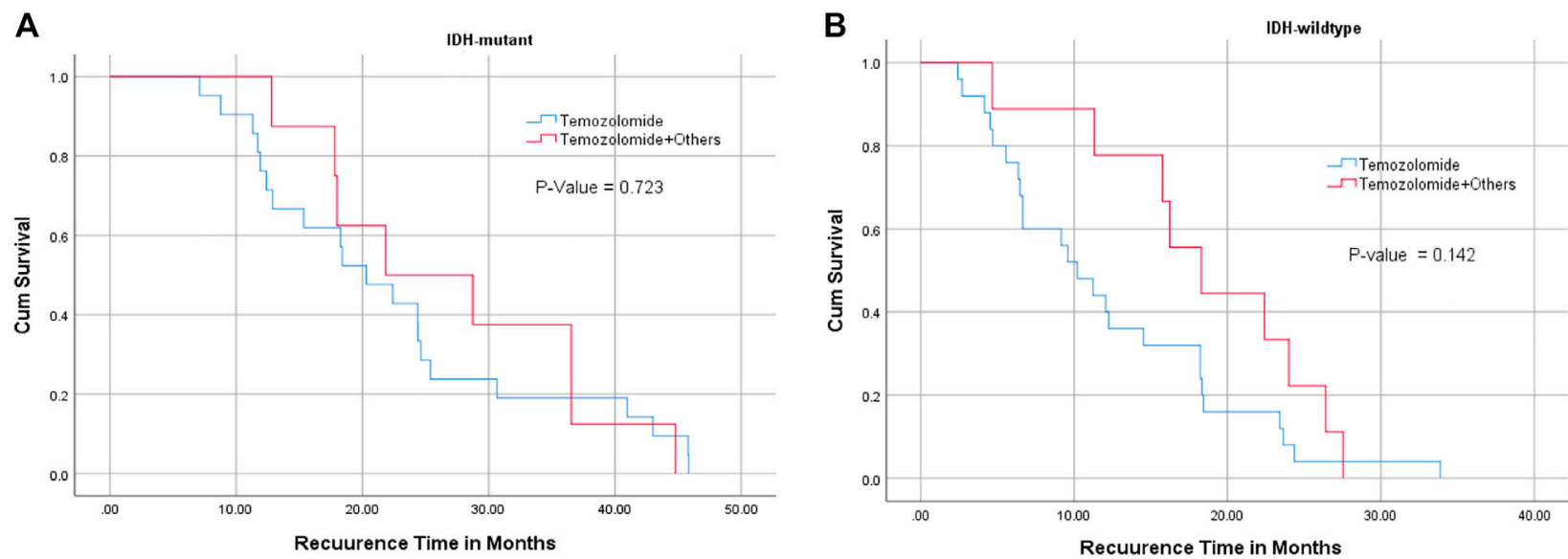

FIGURE 3 | Relationship between IDH1 mutation status ((A) : IDH mutant, (B): IDH wildtype), chemotherapy, and recurrence-free interval. TMZ alone or combined with chemotherapies did not significantly affect tumor recurrence interval in IDH1 mutant and wildtype glioblastoma patients.

IDH1 status (Table 3). However, a difference was clearly seen among glioblastoma patients with unmethylated MGMT promoter who were treated with radiation vs. those who received chemoradiotherapy $(p$-value $=0.031)$ (Figure $2 \mathbf{2 B})$. Among patients with MGMT promoter methylation, there was no significant difference in tumor recurrence interval regardless of the type adjuvant therapy.

\section{Relationship of IDH1 Mutation or MGMT Promoter Methylation and the Type of Chemotherapy With Recurrence-Free Interval in Glioblastoma Patients}

There was no significant relationship between the type of chemotherapy and recurrence-free interval in IDH1 mutant or 
TABLE 4 | The association between IDH1 mutation and MGMT methylation status with recurrence-free interval within 3 years among glioblastoma patients treated with radiotherapy or chemoradiotherapy.

\begin{tabular}{|c|c|c|c|c|c|c|}
\hline & \multirow[b]{2}{*}{ Period in months } & \multirow[b]{2}{*}{ Number risk at end of interval } & \multirow[b]{2}{*}{ Number of cases recurred } & \multirow[b]{2}{*}{ Recurrence free \% } & \multicolumn{2}{|c|}{$95 \% \mathrm{Cl}$} \\
\hline & & & & & Lower & Upper \\
\hline \multicolumn{7}{|l|}{ IDH1 mutant } \\
\hline Radiation & 12 & 2 & 1 & $66.7 \%$ & $30.0 \%$ & $100.0 \%$ \\
\hline Radiation & 24 & 2 & 0 & $66.7 \%$ & $30.0 \%$ & $100.0 \%$ \\
\hline Chemoradiotherapy & 12 & 24 & 5 & $82.8 \%$ & $70.1 \%$ & $97.7 \%$ \\
\hline Chemoradiotherapy & 24 & 13 & 11 & $44.8 \%$ & $29.9 \%$ & $67.1 \%$ \\
\hline \multicolumn{7}{|l|}{ IDH1 wildtype } \\
\hline Radiation & 12 & 6 & 11 & $35.3 \%$ & $18.5 \%$ & $67.2 \%$ \\
\hline Radiation & 24 & 3 & 3 & $17.6 \%$ & $6.3 \%$ & $49.3 \%$ \\
\hline Chemoradiotherapy & 12 & 19 & 14 & $57.6 \%$ & $43.0 \%$ & $77.2 \%$ \\
\hline Chemoradiotherapy & 24 & 6 & 13 & $15.2 \%$ & $6.8 \%$ & $34.0 \%$ \\
\hline \multicolumn{7}{|l|}{ MGMT methylation } \\
\hline Radiation & 12 & 1 & 0 & $100.0 \%$ & $100.0 \%$ & $100.0 \%$ \\
\hline Radiation & 24 & 1 & 0 & $100.0 \%$ & $100.0 \%$ & $100.0 \%$ \\
\hline Chemoradiotherapy & 12 & 9 & 1 & $90.0 \%$ & $73.2 \%$ & $100.0 \%$ \\
\hline Chemoradiotherapy & 24 & 7 & 2 & $70.0 \%$ & $46.7 \%$ & $100.0 \%$ \\
\hline Chemoradiotherapy & 36 & 4 & 3 & $40.0 \%$ & $18.7 \%$ & $85.5 \%$ \\
\hline \multicolumn{7}{|l|}{ Unmethylated MGMT } \\
\hline Radiation & 12 & 1 & 1 & $50.0 \%$ & $12.5 \%$ & $100.0 \%$ \\
\hline Radiation & 24 & 1 & 0 & $50.0 \%$ & $12.5 \%$ & $100.0 \%$ \\
\hline Chemoradiotherapy & 12 & 15 & 4 & $78.9 \%$ & $62.6 \%$ & $99.6 \%$ \\
\hline Chemoradiotherapy & 24 & 6 & 9 & $31.6 \%$ & $16.3 \%$ & $61.2 \%$ \\
\hline Chemoradiotherapy & 36 & 3 & 3 & $15.8 \%$ & $5.6 \%$ & $44.6 \%$ \\
\hline \multirow[t]{2}{*}{ Levels } & & & & & \multicolumn{2}{|c|}{$95 \% \mathrm{Cl}$} \\
\hline & Time & Number risk at end of interval & Number of cases recurred & Recurrence free \% & Lower & Upper \\
\hline \multicolumn{7}{|c|}{ Unmethylated MGMT IDH1 mutant } \\
\hline Temozolomide & 12 & 9 & 4 & $69.2 \%$ & $48.2 \%$ & $99.5 \%$ \\
\hline Temozolomide & 24 & 4 & 5 & $30.8 \%$ & $13.6 \%$ & $69.5 \%$ \\
\hline Temozolomide & 36 & 2 & 2 & $15.4 \%$ & $4.3 \%$ & $55.0 \%$ \\
\hline Temozolomide + Others & 12 & 6 & 0 & $100.0 \%$ & $100.0 \%$ & $100.0 \%$ \\
\hline Temozolomide + Others & 24 & 2 & 4 & $33.3 \%$ & $10.8 \%$ & $100.0 \%$ \\
\hline Temozolomide + Others & 36 & 1 & 1 & $16.7 \%$ & $2.8 \%$ & $99.7 \%$ \\
\hline \multicolumn{7}{|c|}{ Unmethylated MGMT IDH1 wildtype } \\
\hline Temozolomide & 12 & 7 & 7 & $50.0 \%$ & $29.6 \%$ & $84.4 \%$ \\
\hline Temozolomide & 24 & 2 & 5 & $14.3 \%$ & $4.0 \%$ & $51.5 \%$ \\
\hline Temozolomide + Others & 12 & 3 & 0 & $100.0 \%$ & $100.0 \%$ & $100.0 \%$ \\
\hline Temozolomide + Others & 24 & 1 & 3 & $0.0 \%$ & $\mathrm{Na}$ & $\mathrm{Na}$ \\
\hline
\end{tabular}

Cl: confidence interval; Na, not applicable.

wildtype glioblastoma cases (Figure 3) (Table 3). However, patients with MGMT promoter methylation showed significant differences in the recurrence-free interval. In patients with unmethylated MGMT promoter, those treated with TMZ and other chemotherapeutic agents showed late recurrence compared with those who were treated with TMZ alone $(p$-value $=0.014)$ (Tables 3-5).

\section{Recurrence-Free Intervals Between Different Groups}

IDH1-mutant cases treated with only radiotherapy were $66.7 \%$ recurrence free at 12 months of treatment while IDH1-mutant cases who received chemoradiotherapy were $82 \%$ recurrence free at 12 months (Table 4). This clarified that chemoradiotherapy had a better beneficial outcome. In IDH1-wildtype glioblastoma cases treated with chemoradiotherapy showed $57.6 \%$ recurrence free in the $12^{\text {th }}$ month, which is relatively higher than what was observed in IDH1-wildtype cases that received only radiation (35.3\%), however; slightly opposite behavior was observed at 2 years. Another example, cases with MGMT promoter methylation treated with chemoradiotherapy showed recurrence free of $90 \%, 70 \%$ and followed by $40 \%$ at 1,2 , and 3 years, respectively. On the other hand, cases with unmethylated MGMT treated with chemoradiotherapy had recurrence free of $(78.9,31.6$, and $15.8 \%)$ at 1,2 , and 3 years, respectively. This interpretation was quite clear also for the type of chemotherapies used in the treatment of glioblastoma (See Table 4). 
TABLE 5 | The association between IDH1-mutation, MGMT methylation and recurrence-free interval (days) in patients receiving TMZ with additional adjuvant chemotherapies.

\begin{tabular}{|c|c|c|c|}
\hline IDH1 status & MGMT status & Chemotherapies types & $\mathbf{R I}$ \\
\hline IDH-mutant & MGMT hypermethylation & $\mathrm{TMZ}+$ bevacizumab & 1344 \\
\hline IDH-wildtype & MGMT hypermethylation & $\mathrm{TMZ}+$ bevacizumab & 140 \\
\hline IDH-mutant & MGMT hypermethylation & $\mathrm{TMZ}$ + irenotecan + bevacizumab & 1096 \\
\hline IDH-wildtype & Unmethylated & $\mathrm{TMZ}+$ bevacizumab & 720 \\
\hline IDH-wildtype & MGMT hypermethylation & $\mathrm{TMZ}+$ bevacizumab & 340 \\
\hline IDH-wildtype & MGMT hypermethylation & $\mathrm{TMZ}+$ bevacizumab & 672 \\
\hline IDH-wildtype & MGMT hypermethylation & $\mathrm{TMZ}+$ irenotecan + bevacizumab & 792 \\
\hline IDH-mutant & Unmethylated & $\mathrm{TMZ}+$ lomustine + bevacizumab & 862 \\
\hline IDH-wildtype & Unmethylated & TMZ + bevacizumab & 487 \\
\hline IDH-mutant & Unmethylated & $\mathrm{TMZ}+$ bevacizumab & 540 \\
\hline IDH-wildtype & MGMT hypermethylation & $\mathrm{TMZ}$ + lomustine + bevacizumab & 473 \\
\hline IDH-wildtype & MGMT hypermethylation & $\mathrm{TMZ}+$ bevacizumab & 826 \\
\hline IDH-mutant & Unmethylated & $\mathrm{TMZ}+$ bevacizumab & 1096 \\
\hline IDH-mutant & Unmethylated & $\mathrm{TMZ}+$ lomustine & 655 \\
\hline IDH-wildtype & Unmethylated & TMZ + bevacizumab + etoposide & 1004 \\
\hline IDH-wildtype & Unmethylated & TMZ + bevacizumab & 549 \\
\hline IDH-mutant & Unmethylated & $\mathrm{TMZ}+$ bevacizumab & 534 \\
\hline
\end{tabular}

${ }^{*} R l$ : Recurrence interval.

\section{DISCUSSION}

Glioblastoma is the most aggressive primary malignant brain tumor in adults [1]. Both primary and secondary glioblastomas are pathologically indistinguishable, but they vary at the molecular level [2]. The disease remains fatal although the palliative treatment modalities given to the patients [2, 3]. These treatments are currently dependent on several molecular markers including IDH1 mutation and MGMT-gene promoter methylation. Despite the controversy on the impact of these biomarkers, they are still considered prognostic factors in patients with glioblastoma. While wildtype $I D H 1$ is present in $90 \%$ of primary glioblastomas [1], mutant IDH1 is common in secondary glioblastomas and associated with increased patient survival [4, 6]. Epigenetic-mediated silencing of the MGMT gene in glioblastoma by promoter methylation has also been shown to correlate with better OS. Use of IDH1 combined with MGMT promoter as a stratification factor seems appropriate in clinical trials for the treatment of patients with secondary glioblastoma [6].

In the present analysis, we evaluated the impact of MGMT promoter methylation as well as IDH1 mutation status on recurrence-free interval (the period from beginning of adjuvant therapy after surgical resection to the possible first date of recurrence) in patients with glioblastoma. We used recurrence-free interval (RI) rather than OS as RI is more accurate and also doesn't provide false predictability in the outcome of glioblastoma patients.

Because our results found that there was no significant relationship between IDH1 mutation and MGMT promoter methylation, both factors are considered prognostically independent (Table 2). This relationship can be considered significant if the age factor was included in the prognostic analysis. For example, we found that more glioblastoma patients younger than 50 years old with IDH1 mutant tumors had tumor recurrence after one year of treatment compared with those with $I D H 1$ wildtype (Figure 2A; Table 3).
The impact of MGMT promoter methylation status in patients with glioblastoma also showed controversial results. Hegi et al. showed that glioblastomas with methylated MGMT promoters were more sensitive to chemotherapeutic agents, including TMZ, resulting in an OS benefit for these patients [9]. Dunn et al showed that a greater extent of methylation was associated with significantly longer OS [10]. Other studies in anaplastic gliomas have shown that MGMT promoter methylation status did not only influence patient outcome after chemotherapy but also impacted outcome after radiotherapy and may therefore be prognostic rather than predictive [11]. In contrast, other studies revealed that $M G M T$ promoter methylation was not associated with better OS and progression-free survival; both endpoints were comparable in patients with expressed MGMT and those with MGMT silencing. The studies by Costa et al. and Park et al. on glioblastoma patients treated with TMZ-based chemoradiation revealed that MGMT promoter methylation was not associated with improved outcome $[12,13]$. Our study found that MGMT promoter methylation was not associated with a significant change in patient outcome. However, glioblastomas with unmethylated MGMT promoter, who received chemoradiotherapy mainly TMZ therapy, had a late tumor recurrence (Figure 2B; Table 3). Hence, MGMT promoter methylation in glioblastoma cannot be considered as a significant factor for long survival.

The relationships between IDH1 mutation and MGMT promoter methylation with the type of chemotherapies were also assessed independently with tumor recurrence interval. Our results found that glioblastoma cases, regardless of IDH1 status, that were treated with TMZ or TMZ and additional chemotherapeutic agents did not show any difference in tumor recurrence interval (Figure 3). This was completely different among cases in which MGMT methylation was the main independent factor. Although methylated MGMT promoter with IDH1 mutant glioblastoma cases showed clinically significant results, the cases with unmethylated MGMT 
promoter and IDH1 mutation treated with combined chemotherapies had significantly late tumor recurrence. These cases were mostly IDH1 mutant (Table 4). This means that glioblastoma with wildtype IDH1 and unmethylated MGMT promoter should be treated aggressively with combined chemotherapies (TMZ plus other chemotherapies) to improve overall survival. Two other studies also found conflicted findings. Combs et al. analyzed a group of 160 patients with glioblastoma treated with radiotherapy and TMZ for the impact of MGMT promoter methylation and IDH1 mutation. Unexpectedly, OS was no longer among the group of methylated MGMT promoter compared with patients without MGMT promoter methylation [17]. In contrast, Millward et al. demonstrated that the combination of methylated MGMT and IDH1 mutation was associated with considerably longer OS and PFS in a series of chemoradiotherapy-treated glioblastoma tumors [14]. Alassiri et al. also found a positive impact of both MGMT methylation and $I D H 1$ mutation on the overall survival of Saudi patients with glioblastoma [18] but the study did not relate the findings with other treatment modalities. Another recent study by Pandith et al investigated the relationship between IDH1/IDH2 mutations and MGMT methylation in patients receiving chemotherapies in different types of malignant gliomas (astrocytomas and oligodendrogliomas). Compared to our study, the only treatment modality used in their study was TMZ chemotherapy while IDH1/2 sequencing was the primary method to detect mutation. Using IDH1/IDH2 sequencing from fresh tissue nowadays are somewhat difficult either because of limited facilities or because the high cost of sequencing [19]. Our results concluded that IDH1 mutation and MGMT gene promoter methylation are considered independent prognostic factors in glioblastoma. Although IDH1 mutant glioblastomas showed late tumor recurrence in patients younger than 50 years of age, the type of treatment modalities may not show additional beneficial outcome. Patients with unmethylated MGMT gene promoter with IDH1 mutation, treated with chemoradiotherapy including TMZ had a late tumor recurrence while glioblastomas with wildtype IDH1 and unmethylated MGMT gene promoter should be treated aggressively with radiotherapy and combined chemotherapies to delay tumor recurrence.

One limitation must be acknowledged in our study, that the total number of cases analyzed for MGMT promoter methylation and IDH1 mutation are low. Despite this limitation, our report is the first study in Saudi Arabia that correlates these molecular biomarkers with recurrence-free interval in totally resected glioblastomas, reflecting the impact of adjuvant therapies as well as the specific type of chemotherapies on patient outcome.

\section{CONCLUSION}

This is the first paper to describe the role of IDH1 mutation and MGMT promoter methylation status in terms of overall survival and recurrence-free survival by patients group treated with different therapeutical modalities in Saudi Arabia. The impact of IDH mutations and MGMT promoter methylation on OS and PFS is extensively described in the literature. Our results highlighted the impact of these molecular biomarkers on different treatment modalities including radiotherapy and chemotherapies on glioblastoma outcome. Additional molecular studies and raising the sample size could increase the value of the investigation.

\section{DATA AVAILABILITY STATEMENT}

The data that support the findings of this study are available from the corresponding author upon request.

\section{ETHICS STATEMENT}

The studies involving human participants were reviewed and approved by the National biomedical Ethics Committee of King Abdulaziz University (HA-02-J-008) which comply with the guidelines of the "System of ethics of research" prepared by the King Abdulaziz City for Science and Technology, and approved by Royal Decree No. M/59 on 24 August 2010. The patients/participants provided their written informed consent to participate in this study.

\section{AUTHOR CONTRIBUTIONS}

MK, idea, IRB submission, writing, study design and data analysis; NB, Statistical analysis; SB, data provider, writing, and analysis; BA, study design, writing, and editing; YM, data entry, tissue collection, and writing; $\mathrm{AB}$, data entry, and tissue collection; RS, data entry, tissue collection, and writing; TA, data provider, writing, and analysis; NA, records collection, and data interpretation; MB, tissue processing, and IHC; AS, data provider, and writing; $\mathrm{AL}$, data provider, and IRB submission.

\section{CONFLICT OF INTEREST}

The authors declare that the research was conducted in the absence of any commercial or financial relationships that could be construed as a potential conflict of interest.

\section{ACKNOWLEDGMENTS}

We thank all patients, physicians, clinical trial nurses, laboratory staff, and technicians for their participation in this study. 


\section{REFERENCES}

1. Louis DN, Ohgaki H, Wiestler OD, and Cavenee WK. World Health Organization Histiological Classification of Tumours of the Central Nervous System. France: International Agency for Research on Cancer (2016).

2. Ohgaki H, Dessen P, Jourde B, Horstmann S, Nishikawa T, Di Patre PL, et al. Genetic pathways to glioblastoma. Cancer Res (2004) 64(19):6892-9. doi:10.1158/0008-5472.can-04-1337

3. Stupp R, Hegi ME, Mason WP, Van den Bent MJ, Taphoorn MJB, Janzer RC, et al. (2009) Effects of radiotherapy with concomitant and adjuvant temozolamide versus radiotherapy alone on survival in glioblastoma in a randomized phase III study: 5-year analysis of the EORTC-NCIC trial. Lancet Oncol 10(5):459-66. doi:10.1016/s1470-2045(09)70025-7

4. Hartmann C, Meyer J, Balss J, Capper D, Mueller W, Christians A, et al. Type and frequency of IDH1 and IDH2 mutations are related to astrocytic and oligodendroglial differentiation and age: a study of 1,010 diffuse gliomas. Acta Neuropathol (2009) 118:469-74. doi:10.1007/s00401-009-0561-9

5. Capper D, Weĩ̃Ÿert S, Balss JR, Habel A, Meyer J, Jãger D, et al. Characterization of $\mathrm{R} 132 \mathrm{H}$ mutation-specific IDH1 antibody binding in brain tumors. Brain Pathol (2010) 20:245-54. doi:10.1111/j.1750-3639. 2009.00352.x

6. Nobusawa S, Watanabe T, Kleihues $\mathrm{P}$, and Ohgaki H. IDH1 mutations as molecular signature and predictive factor of secondary glioblastomas. Clin Cancer Res (2009) 15(19):6002-7. doi:10.1158/1078-0432.ccr-09-0715

7. Pegg AE. Repair of O6-alkylguanine by alkyltransferases. Mutat Research/ Reviews Mutat Res (2000) 462:83-100. doi:10.1016/s1383-5742(00)00017-x

8. Ludlum DB. DNA alkylation by the haloethylnitrosoureas: nature of modifications produced and their enzymatic repair or removal. Mutat Research/Fundamental Mol Mech Mutagenesis (1990) 233:117-26. doi:10. 1016/0027-5107(90)90156-x

9. Hegi ME, Diserens AC, Gorlia T, Hamou MF, de Tribolet N, Weller M, et al. MGMTGene silencing and benefit from temozolomide in glioblastoma. N Engl J Med (2005) 352:997-1003. doi:10.1056/nejmoa043331

10. Dunn J, Baborie A, Alam F, Joyce K, Moxham M, Sibson R, et al. Extent of MGMT promoter methylation correlates with outcome in glioblastomas given temozolomide and radiotherapy. Br J Cancer (2009) 101:124-31. doi:10.1038/ sj.bjc. 6605127

11. Wick W, Hartmann C, Engel C, Stoffels M, Felsberg J, Stockhammer F, et al. NOA-04 randomized phase III trial of sequential radiochemotherapy of anaplastic glioma with procarbazine, lomustine, and vincristine or temozolomide. Jco (2009) 27:5874-80. doi:10.1200/jco.2009.23.6497
12. Costa BM, Caeiro C, Guimarães I, Martinho O, Jaraquemada T, Augusto I, et al. Prognostic value of MGMT promoter methylation in glioblastoma patients treated with temozolomide-based chemoradiation: a Portuguese multicentre study. Oncol Rep (2010) 23:1655-62. doi:10.3892/or_00000808

13. Park C-K, Park S-H, Lee S-H, Kim C-Y, Kim D-W, Paek SH, et al. Methylation status of the MGMT gene promoter fails to predict the clinical outcome of glioblastoma patients treated with ACNU plus cisplatin. Neuropathology (2009) 29:443-9. doi:10.1111/j.1440-1789.2008.00998.x

14. Millward CP, Brodbelt AR, Haylock B, Zakaria R, Baborie A, Crooks D, et al. The impact of MGMT methylation and IDH-1 mutation on long-term outcome for glioblastoma treated with chemoradiotherapy. Acta Neurochir (2016) 158:1943-53. doi:10.1007/s00701-016-2928-8

15. Esteller M, Hamilton SR, Burger PC, Baylin SB, and Herman JG. Inactivation of the DNA repair gene O6-methylguanine-DNA methyltransferase by promoter hypermethylation is a common event in primary human neoplasia. Cancer Res (1999) 59:793-7.

16. Panagopoulos I, Gorunova L, Leske H, Niehusmann P, Johannessen LE, Staurseth J, et al. Pyrosequencing Analysis of MGMT promoter methylation in meningioma. Cancer Genomics Proteomics (2018) 15: 379-85. doi:10.21873/cgp.20096

17. Combs S, Reikan S, Wick W, Abdollah A, Deimling AV, Debus J, et al. Prognostic significance of IDH1 and MGMT in patients with glioblastoma: one step forward, and one step back. Radiat Oncol (2011) 6: 115. doi:10.1186/1748-717x-6-115

18. Alassiri AH, Alkhaibary A, Al-Sarheed S, Alsufani F, Alharbi M, Alkhani A, et al. O 6 -methylguanine-DNA methyltransferase promoter methylation and isocitrate dehydrogenase mutation as prognostic factors in a cohort of Saudi patients with glioblastoma. Ann Saudi Med (2019) 39(6):410-6. doi:10.5144/ 0256-4947.2019.410

19. Pandith AA, Qasim I, Baba SM, Koul A, Zahoor W, Afroz D, et al. Favorable role of IDH1/2 mutations aided with MGMT promoter gene methylation in the outcome of patients with malignant glioma. Future Sci OA (2020) 7(3): FSO663. doi:10.2144/fsoa-2020-0057

Copyright (c) 2021 Kurdi, Shafique Butt, Baeesa, Alghamdi, Maghrabi, Bardeesi, Saeedi, Al-Sinani, Alghanmi, Bari, Samkari and Lary. This is an open-access article distributed under the terms of the Creative Commons Attribution License (CC BY). The use, distribution or reproduction in other forums is permitted, provided the original author(s) and the copyright owner(s) are credited and that the original publication in this journal is cited, in accordance with accepted academic practice. No use, distribution or reproduction is permitted which does not comply with these terms. 